\title{
CODEXS: A New Multidimensional Index to Better Predict Frequent COPD Exacerbators with Inclusion of Depression Score
}

This article was published in the following Dove Press journal: International Journal of Chronic Obstructive Pulmonary Disease

\author{
Dingding Deng ${ }^{1} * *$ \\ Aiyuan Zhou ${ }^{2-4, *}$ \\ Ping Chen ${ }^{2-4}$ \\ Qingcui Shuang' \\ 'Department of Respiratory Medicine, \\ First Affiliated People's Hospital of \\ Shaoyang College, Shaoyang, Hunan \\ 42200 I, People's Republic of China; \\ ${ }^{2}$ Department of Respiratory and Critical \\ Medicine, Second Xiangya Hospital, \\ Central South University, Changsha, \\ Hunan 4I00II, People's Republic of \\ China; ${ }^{3}$ Respiratory Disease Research \\ Unit, Central South University, Changsha, \\ Hunan 4I00II, People's Republic of \\ China; ${ }^{4}$ Respiratory Disease Diagnosis \\ and Treatment Center, Central South \\ University, Changsha, Hunan 4I00II, \\ People's Republic of China
}

*These authors contributed equally to this work
Correspondence: Qingcui Shuang Department of Respiratory Medicine, First Affiliated People's Hospital of Shaoyang College, West Side of Dongfeng Pedestrian Street, Shuangqing District,

Shaoyang, Hunan 42200I, People's

Republic of China

Tel +86 I36 07398125

Fax +86 0739523444 I

Email shuangqingcui@sina.com
Purpose: Depression is reported in association with chronic obstructive pulmonary disease (COPD). However, to date, no multidimensional indices have taken depression into consideration to predict COPD patients' prognosis. This study aimed to determine whether a new multidimensional index named CODEXS, based on comorbidities, airflow obstruction, dyspnea, previous exacerbation and depression assessed by Self-Rating Depression Scale (SDS), could predict 1-year exacerbations.

Methods: This was a prospective study, patients with stable COPD were used to develop CODEXS at the first visit, and followed up in the 3rd, 6th, and 12th months. After the last visit, patients were divided into frequent and infrequent exacerbators. Another cohort of COPD patients was used for validation. The SDS scoring system in the multidimensional indices ranged from 0 to 4 based on the modified SDS value, representing no depression (25-39 [0], 40-49 [1]), mild depression (50-59), moderate depression (60-69), and severe depression ( $\geq 70$ ). Comorbidity, dyspnea, airflow obstruction, and severe exacerbations were calculated according to CODEX thresholds.

Results: Two sets of 105 and 107 patients were recruited in the development and validation cohorts, respectively. Depression was demonstrated as an independent risk factor for frequent exacerbators (odds ratio $(\mathrm{OR})=1.14,95 \%$ confidence interval $(\mathrm{CI})=1.06-1.23, P<0.001$ ). The prevalence of depression in frequent exacerbators $(35.09 \%)$ was higher than that in infrequent exacerbators. CODEXS was significantly associated with exacerbation $(\mathrm{OR}=2.91 ; 95 \% \mathrm{CI}, 1$.$89-4.48, p<0.001$ ). Receiver operating characteristic (ROC) curve comparison showed that CODEXS was superior to BODEX(BMI, airflow obstruction, dyspnea, previous exacerbation), BODE (BMI, airflow obstruction, dyspnea, exercise), and updated ADO (age, dyspnea, and airflow obstruction) indices, confirmed by the validation cohort with sensitivity at $85.94 \%$ and specificity at $76.74 \%$.

Conclusion: Depression is an independent risk factor for COPD exacerbation. CODEXS is a useful predictor for predicting frequent exacerbators within 1 year and is superior to other previously published indices.

Keywords: COPD, depression, Self-Rating Depression Scale, exacerbation, CODEXS

\section{Introduction}

Chronic obstructive pulmonary disease (COPD) is a preventable and treatable disease which is characterized by persistent respiratory symptoms and persistent airflow limitation, and is currently the fourth leading cause of mortality worldwide. Acute exacerbation of COPD (AECOPD) is an event defined as acute worsening of respiratory symptoms that result in additional therapy. ${ }^{1}$ It can accelerate deterioration of the disease, ${ }^{2,3}$ lead to 
a heavy economic burden, ${ }^{4}$ and increase mortality. ${ }^{5}$ Recently, there is an increasing recognition that COPD is a complex and heterogeneous disease. ${ }^{6}$ In the review we published in $2017,{ }^{7}$ we concluded that there are different phenotypes in the exacerbation stage, one of which is called the "frequent exacerbator" phenotype. The frequency of AECOPD is variable among patients; a proportion of COPD patients seldom exacerbate, but others do so frequently. ${ }^{8}$ As such, identifying those patients who are at high risk and intervening in a timely manner is a clinical priority. Many variables are proved to be related to outcome in patients with COPD; the most recognized variables are postbronchodilator $\mathrm{FEV}_{1}$, dyspnea, and historical exacerbations. $^{5,9}$ GOLD (Global Initiative for Chronic Obstructive Lung Disease) recommendations have taken all these variables into consideration for grading COPD severity. ${ }^{10}$ The use of multidimensional indices, such as CODEX (comorbidity, airflow obstruction, dyspnea, previous severe exacerbations), ${ }^{11}$ BODE (BMI, airflow obstruction, dyspnea, and exercise capacity), ${ }^{12}$ BODEX (BMI, airflow obstruction, dyspnea, and previous severe exacerbations), and updated ADO (age, dyspnea, and airflow obstruction), ${ }^{13}$ have improved the prognostic capacity beyond individual variables.

Depression is a recognized complication of many chronic diseases, including COPD. ${ }^{14,15}$ Some studies have found that depression is common in COPD, with the prevalence increasing with the severity of disease, from $19.6 \%$ in the mild-to-moderate group to $25 \%$ in the severe group. ${ }^{14}$ Patients with severe COPD are at increased risk of developing depression to increase the frequency of exacerbations. ${ }^{16}$ The Self-Rating Depression Scale (SDS) is a 20 -item self-report questionnaire that can be used to screen for and assess depression. ${ }^{17}$ It consists of four common characteristics of depression: the pervasive effect, physiological equivalents, other disturbances, and psychomotor activities. There are 10 positive and 10 negative questions. Each question is scored on a scale of 1-4 (a little of the time, some of the time, a good part of the time, most of the time), and the total score ranges from 20 to 80 . The raw score is then converted to an index score by dividing the raw score by the maximum score (80) and either expressing this as a decimal or multiplying by 100 to express it as a whole number with an index score range of 25 to 100 . An index score of 25 to 49 indicates no depression, 50-59 indicates mild to moderate depression, 60-69 indicates moderate to severe depression, and a score of 70 or over indicates severe depression. ${ }^{18}$
In this study, we investigated the relationship between depression and the outcome of patients with COPD, and determined whether the inclusion of SDS could improve the predictive value of the previously established multidimensional indices ADO, BODEX, BODE, and CODEX.

\section{Patients and Methods}

This research was approved by the local Ethics Committee of the First Affiliated People's Hospital of Shaoyang College and conducted in accordance with the Declaration of Helsinki and its amendments, and all subjects gave written informed consent to participate in the study. The study was registered in the Chinese Clinical Trial Registry (ChiCTR-ROC-16009087; http://www.chictr.org.cn/).

\section{Study Subjects}

Two cohorts of patients diagnosed with stable COPD were recruited at the outpatient department of the First Affiliated People's Hospital of Shaoyang College during different time periods from September 2015 to August 2018. One was used to develop updated multidimensional indices which included the SDS score for predicting frequent exacerbators; the other was collected to validate the new multidimensional indices. The diagnosis of COPD was based on the latest GOLD document. ${ }^{1}$ Patients with a history of diagnosis of asthma, bronchiectasis, lung fibrosis, upper airway obstruction, or tuberculosis were excluded. Patients in the exacerbation stage were excluded. Patients who could not understand the SDS questionnaire or refused to complete the questionnaire were also excluded.

\section{Study Design}

This was a prospective study. All the patients in this study accepted four visits. At the first visit, all participants signed a written informed consent and provided the following information: age, sex, smoking history, career, education, comorbidities, BMI, COPD assessment test (CAT), modified Medical Research Council Dyspnea Scale (mMRC), Clinical COPD Questionnaire (CCQ), SDS, 6 min walk distance, history of exacerbations in the previous year, and lung function. Before using the SDS report, the researchers explained the content of the questionnaire to the patients in detail, making sure the patients understand the meaning of each item. Then, the patients were asked to rate each of the 20 items as to how it applied to them at the time of testing. If the patients forgot the meaning of any item in completing the form, the researcher could explain again without any intervention and guidance. Comorbidity was quantified according to the Charlson index. ${ }^{19} \mathrm{CAT}$, mMRC, and CCQ were collected as described previously. ${ }^{20,21}$ The 6-mins 
walk test was performed according to the guideline. ${ }^{22}$ The BODE, BODEX, CODEX, and ADO scores were calculated based on previous studies. ${ }^{11-13,23}$ The SDS score included in the multidimensional indices ranged from 0 to 4 based on the severity of depression, representing 25-39, 40-49, 50-59, $60-69$, and $\geq 70$, respectively. Then, the SDS value was added into BODE, BODEX, ADO, and CODEX to develop the updated multidimensional indices, named BODES, BODEXS, ADOS, and CODEXS, respectively. After the baseline visit, every participant was followed up in the 3rd, 6th, and 12th months, respectively, to record the occurrence of exacerbations and the treatment therapy during 1 year of follow-up.

\section{Definition of Exacerbations}

Exacerbations were diagnosed based on event-based definition: worsening or new onset of any respiratory symptoms (cough, sputum volume or purulence, wheezing, or dyspnea) for at least 3 days that led to any of the following situations: 1. requiring prescription change under the guidance of a physician in the outpatient department; 2. requiring hospitalization or an emergency room visit with the diagnosis of AECOPD. Exacerbations separated by $\geq 14$ days were considered distinct events. The frequent exacerbators were those who had at least two exacerbations or one hospitalization per year.

\section{Statistical Analysis}

Adjusted multiple logistic regression models were performed to determine the association between variables and COPD exacerbations. The variables age, gender, BMI, smoking status, exacerbation history, GOLD stage, CCQ, CAT, mMRC, and SDS were taken into consideration. Comparison of mean SDS score and GOLD stage was performed with one-way ANOVA. The Student $t$ test or Mann-Whitney $U$-test was used to compare means difference for continuous variables and the chisquared test was used for categorical variables. All statistical analyses above were performed using SPSS 25 . Receiver operating characteristic (ROC) analysis was used to determine the predictive capacity of different multidimensional indices. Comparisons between the area under the curves (AUC) were made using the Hanley-McNeil test through Medcalc software, and a $P$ value of $<0.05$ was considered statistically significant.

\section{Results}

\section{Patient Demographic Characteristics}

A flow chart of the study is shown in Figure 1. Initially, a total of 180 subjects with a primary diagnosis of COPD were screened. Among them, 57 patients were excluded because of exacerbation ( $n=15)$, asthma $(n=23)$, fibrosis $(\mathrm{n}=9)$, or bronchiectasis $(\mathrm{n}=10)$. Ten patients refused to perform the 6-mins walk test and dropped out. During the 1-year follow-up period, eight patients dropped out: one of them died because of extrapulmonary disease and seven of them could not be reached. Finally, 105 patients were recruited into the final analysis. Demographic and clinical data are presented in Table 1. The mean age of the patients was $67.97 \pm 8.91$ years, with $94.29 \%$ males and $5.71 \%$ females. The mean $\mathrm{FEV}_{1} / \mathrm{FVC}$ and predicted $\mathrm{FEV}_{1} \%$ were $49.90 \pm 9.33 \%$ and $49.56 \pm 15.42 \%$. The average mMRC, CAT, CCQ, and SDS scores were $1.43 \pm 0.85,13.63 \pm 7.10$, $3.11 \pm 3.88$, and $40.59 \pm 14.42$, respectively. The average number of exacerbations was $1.10 \pm 1.25$ per year.

\section{Depression Is an Independent Risk Factor for COPD Exacerbation}

After 1 year of follow-up, 57 (54.29\%) patients were grouped as frequent exacerbators. A logistic regression model was used to evaluate the prognostic value of each variable. Univariate analysis showed that several factors, including $\mathrm{FEV}_{1} \%$, CAT, mMRC, SDS, comorbidities, treatment, and history of exacerbations contributed to frequent exacerbations (Table 2). These factors except CAT were then further analyzed by multivariate analysis. The multivariate model showed that SDS (odds ratio $(\mathrm{OR})=1.14,95 \%$ confidence interval $(\mathrm{CI})=1.06-1.23$, $P<0.001)$ and history of exacerbations (OR $=2.33,95 \%$ $\mathrm{CI}=1.20-4.51, P<0.001)$ were independent indicators of frequent exacerbations; regular treatment $(\mathrm{OR}=0.03,95 \%$ $\mathrm{CI}=0.01-0.14, P<0.001)$ could decrease exacerbation significantly (Table 2 ).

\section{Depression Severity Is Correlated with GOLD Stage and Symptom Severity}

The prevalence of depression in the frequent exacerbators $(35.09 \%)$ was higher than that in infrequent exacerbators (12.5\%) (Figure 2). The SDS score in patients at GOLD stage IV was significantly higher than that in other stages. The average SDS score of patients at GOLD stages I, to IV was $28.33 \pm 2.89,32.73 \pm 10.96,43.50 \pm 11.51$, and 63.91 \pm 8.76 , respectively (Figure 3 ). The SDS score was positively correlated with the GOLD stage (rho $=0.641$, $P<0.001$ ), CAT score (rho $=0.528, P<0.001$ ), and $\mathrm{mMRC}(\mathrm{rho}=0.425, P<0.001)$. 


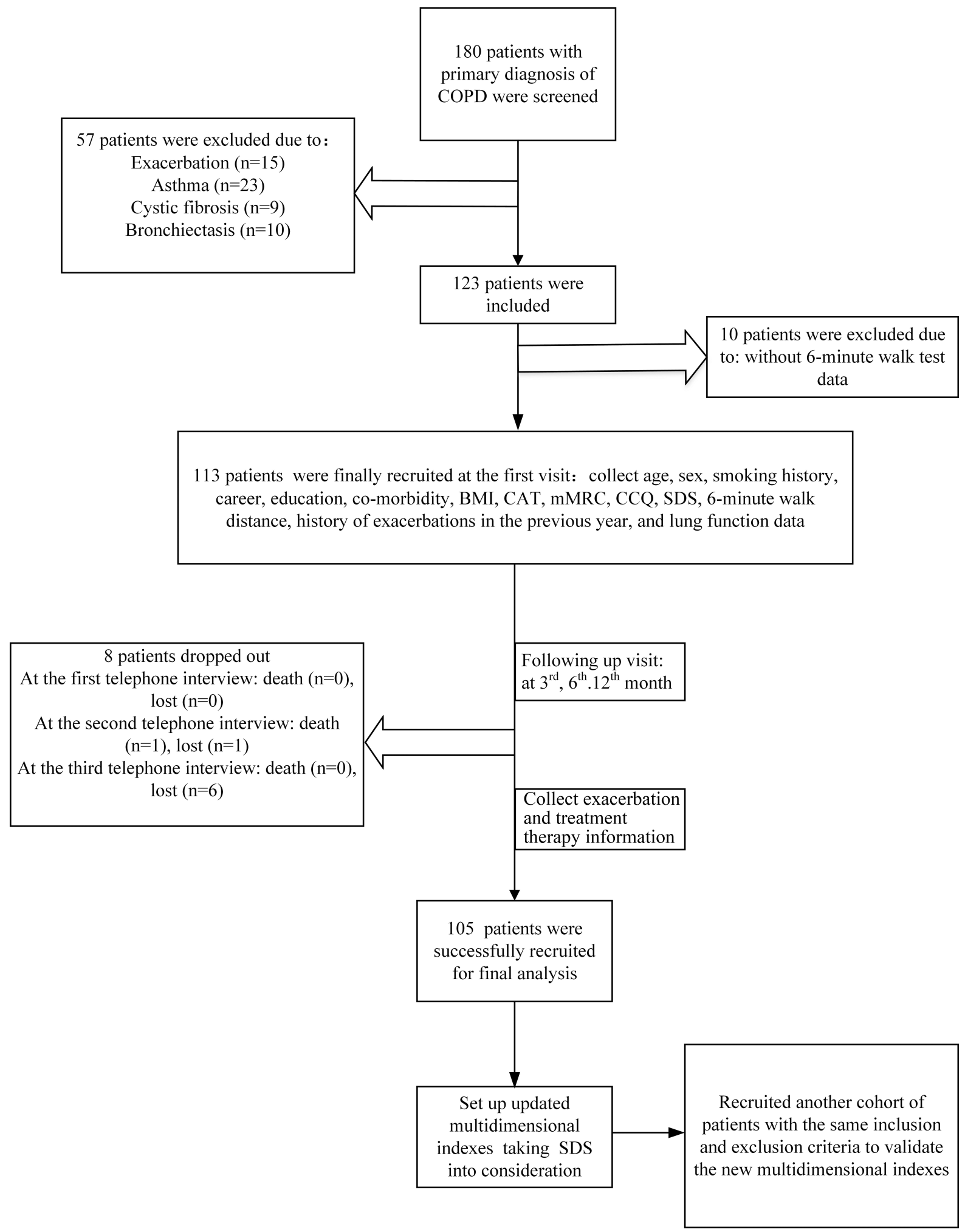

Figure I Study flow chart. A total of I 80 subjects with a primary diagnosis of COPD were screened; 57 patients were excluded because of exacerbation $(n=I 5)$, asthma $(n=23)$, fibrosis $(n=9)$, or bronchiectasis $(n=10)$. Ten patients refused to perform the 6-mins walk test and dropped out. Seven of them could not be reached. During the I-year follow-up period, eight patients dropped out; one of them died because of extrapulmonary disease and seven of them could not be reached. After developing the updated multidimensional indices, another cohort of patients was recruited to validate the indices. 
Table I Demographic and Clinical Characteristics of Patients

\begin{tabular}{|c|c|c|c|c|c|c|}
\hline \multirow[t]{2}{*}{ Variables } & \multirow[t]{2}{*}{ Characteristics } & \multicolumn{2}{|c|}{ Cohort I } & \multicolumn{2}{|c|}{ Cohort 2} & \multirow[t]{2}{*}{$p$} \\
\hline & & $\mathbf{n}$ & $\% /$ Mean \pm SD & $\mathbf{n}$ & $\% /$ Mean \pm SD & \\
\hline Age & & 105 & $67.87 \pm 8.91$ & 107 & $68.48 \pm 8.43$ & 0.61 \\
\hline \multirow[t]{2}{*}{ Sex } & Male & 99 & $94.29 \%$ & 99 & $92.5 \%$ & 0.61 \\
\hline & Female & 6 & $5.71 \%$ & 9 & $7.5 \%$ & \\
\hline BMI & & 105 & $21.70 \pm 5.86$ & 107 & $21.22 \pm 2.28$ & 0.44 \\
\hline \multirow[t]{2}{*}{ Smoking } & Ex-smoker & 14 & $13.33 \%$ & 20 & $18.69 \%$ & 0.29 \\
\hline & Current smoker & 91 & $86.67 \%$ & 87 & $81.31 \%$ & \\
\hline \multicolumn{2}{|l|}{$\mathrm{FEV}_{\mathrm{I}} / \mathrm{FVC}$} & 105 & $49.90 \pm 9.33 \%$ & 107 & $47.38 \pm 9.40 \%$ & 0.68 \\
\hline \multicolumn{2}{|l|}{$\mathrm{FEV}, \%$} & 105 & $49.56 \pm 15.42 \%$ & 107 & $48.31 \pm 16.12 \%$ & 0.57 \\
\hline \multicolumn{2}{|l|}{ CAT } & 105 & $13.63 \pm 7.10$ & l & I & \\
\hline \multicolumn{2}{|l|}{$\mathrm{mMRC}$} & 105 & $\mathrm{I} .43 \pm 0.85$ & 107 & $1.52 \pm 0.93$ & 0.44 \\
\hline \multicolumn{2}{|l|}{ CCQ } & 105 & $3.11 \pm 3.88$ & l & I & \\
\hline \multicolumn{2}{|l|}{ SDS } & 105 & $40.59 \pm 14.42$ & 107 & $41.42 \pm 12.08$ & 0.65 \\
\hline \multirow[t]{4}{*}{ GOLD stage } & Mild & 3 & $2.86 \%$ & 3 & $2.80 \%$ & 0.79 \\
\hline & Moderate & 45 & $42.86 \%$ & 39 & $36.45 \%$ & \\
\hline & Severe & 46 & $43.80 \%$ & 54 & $50.47 \%$ & \\
\hline & Very severe & 11 & $10.48 \%$ & 11 & $10.28 \%$ & \\
\hline \multirow{2}{*}{\multicolumn{2}{|c|}{ Comorbidities }} & 26 & $24.76 \%$ & 39 & $36.45 \%$ & 0.17 \\
\hline & & 79 & $75.24 \%$ & 68 & $63.55 \%$ & \\
\hline \multicolumn{2}{|c|}{6 min walk distance $(m)$} & 105 & $331.72 \pm 91.09$ & l & I & \\
\hline \multirow[t]{2}{*}{ Location } & Urban areas & 72 & $68.57 \%$ & l & I & \\
\hline & Rural areas & 33 & $31.43 \%$ & l & I & \\
\hline \multirow[t]{5}{*}{ Education } & Uneducated & 9 & $8.58 \%$ & l & I & \\
\hline & Primary school & 40 & $38.10 \%$ & l & I & \\
\hline & Junior school & 32 & $30.48 \%$ & I & I & \\
\hline & High school & 16 & $15.24 \%$ & I & 1 & \\
\hline & University & 8 & $7.62 \%$ & l & 1 & \\
\hline \multirow[t]{3}{*}{ Occupation } & Farmer & 42 & $40 \%$ & l & l & \\
\hline & Worker & 38 & $36.19 \%$ & l & l & \\
\hline & Other & 25 & $23.81 \%$ & l & l & \\
\hline \multicolumn{2}{|c|}{ History of exacerbations } & 105 & $1.10 \pm 1.25$ & 107 & $1.15 \pm 0.99$ & 0.42 \\
\hline
\end{tabular}

Abbreviations: $\mathrm{BMI}$, body mass index; $\mathrm{FEV}_{\mathrm{l}}$, forced expiratory volume in I s; FVC, forced vital capacity; CAT, COPD assessment test; mMRC, modified Medical Research Council test; CCQ, Clinical COPD Questionnaire score; SDS, Self-Rating Depression Scale; GOLD, Global Initiative for Chronic Obstructive Lung Disease.

\section{Comparison of the Predictive Value of Different Multidimensional Indices}

The ADO, CODEX, BODE, and BODEX indices could predict the frequent exacerbators effectively: the OR were 2.0, 2.79, 1.91, and 2.17, respectively. Comparison of the ROC curves showed that CODEX had a better predictive capacity for the frequent exacerbators compared with $\mathrm{ADO}$ $(P=0.008)$ and BODEX $(P=0.03)$; the AUC were 0.82, 0.71 , and 0.79 , respectively (Table 3 and Figure 4).
Combined Variables Improve the Predictive Value for Frequent Exacerbators

With respect to the combined variable (SDS value), all indices reached statistical significance for predicting frequent exacerbators (Table 4). ROC curve comparison showed that the inclusion of SDS value improved the predictive capacity of all the previously established indices (ADOS vs ADO, 0.80 [0.71], $P=0.0002$ [Figure 5A]; 
Table 2 Univariate and Stepwise Multivariate Analysis of Risk Factors for Frequent Exacerbators

\begin{tabular}{|c|c|c|c|c|}
\hline & \multicolumn{2}{|c|}{$\begin{array}{l}\text { Univariate } \\
\text { Analysis }\end{array}$} & \multicolumn{2}{|c|}{$\begin{array}{l}\text { Multivariate } \\
\text { Analysis }\end{array}$} \\
\hline & OR & $95 \% \mathrm{Cl}$ & OR & $95 \% \mathrm{Cl}$ \\
\hline Age (years) & 1.0 & $0.96-1.05$ & 0.99 & $0.92-1.06$ \\
\hline Gender (male) & 1.2 & $0.23-6.24$ & - & \\
\hline BMI & 1.02 & $0.95-1.10$ & - & \\
\hline $\mathrm{FEV}_{1} / \mathrm{FVC}$ & 0.94 & $0.89-0.98 *$ & \multicolumn{2}{|c|}{ Not selected } \\
\hline $\mathrm{FEV}, \%$ & 0.94 & $0.91-0.97 *$ & 0.99 & $0.93-1.05$ \\
\hline CAT & I.II & $1.04-1.18^{*}$ & \multicolumn{2}{|c|}{ Not selected } \\
\hline $\mathrm{mMRC}$ & 2.67 & $1.51-4.73 *$ & 1.27 & $0.53-3.05$ \\
\hline CCQ & 1.05 & $0.93-1.18$ & \multicolumn{2}{|l|}{-} \\
\hline SDS & 1.12 & $1.07-1.17^{*}$ & 1.14 & $1.06-1.23 *$ \\
\hline 6 min walk distance & 0.99 & $0.98-1.00$ & - & \\
\hline Location (city) & 0.60 & $0.26-1.37$ & - & \\
\hline Comorbidities & 2.92 & I.15-7.40* & & \\
\hline Job & 1.23 & $0.75-2.01$ & - & \\
\hline History of exacerbations & 1.96 & $1.25-3.09 *$ & 2.33 & $\mid .20-4.5 I^{*}$ \\
\hline Smoking history & 1.22 & $0.40-3.76$ & \multicolumn{2}{|l|}{ - } \\
\hline Standardized treatment & 0.07 & $0.03-0.20 *$ & 0.03 & $0.01-0.14^{*}$ \\
\hline
\end{tabular}

Note: *Means $P<0.05$.

Abbreviations: $\mathrm{BMI}$, body mass index; $\mathrm{FEV}_{1}$, forced expiratory volume in I s; FVC, forced vital capacity; CAT, COPD assessment test; mMRC, modified Medical Research Council test; CCQ, Clinical COPD Questionnaire score; SDS, Self-Rating Depression Scale.

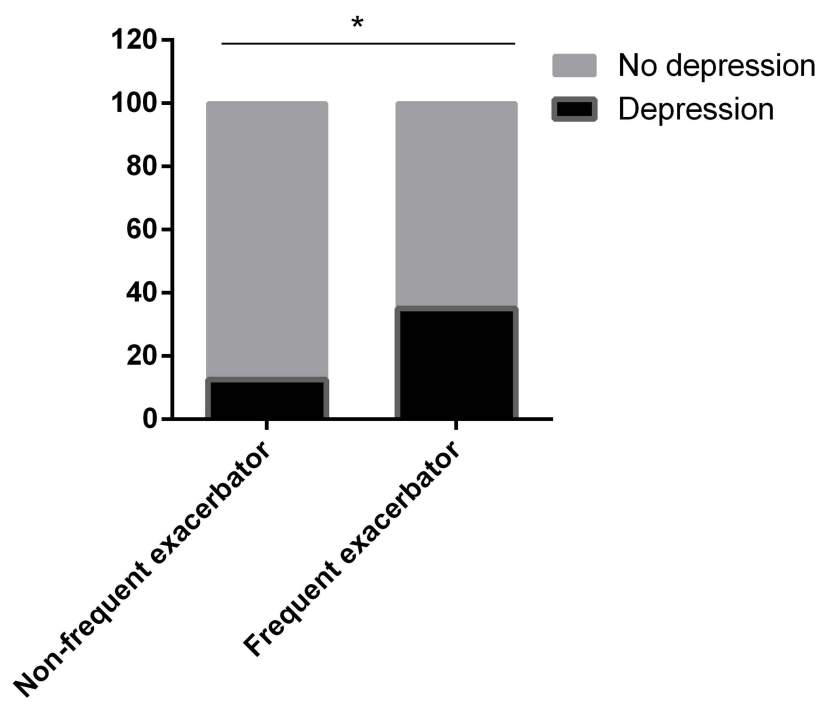

Figure 2 Prevalence of depression in COPD patients. The prevalence of depression in the frequent exacerbators (35.09\%) was higher than that in infrequent exacerbators (12.5\%). *Means $P<0.05$.

Abbreviation: COPD, chronic obstructive pulmonary disease.

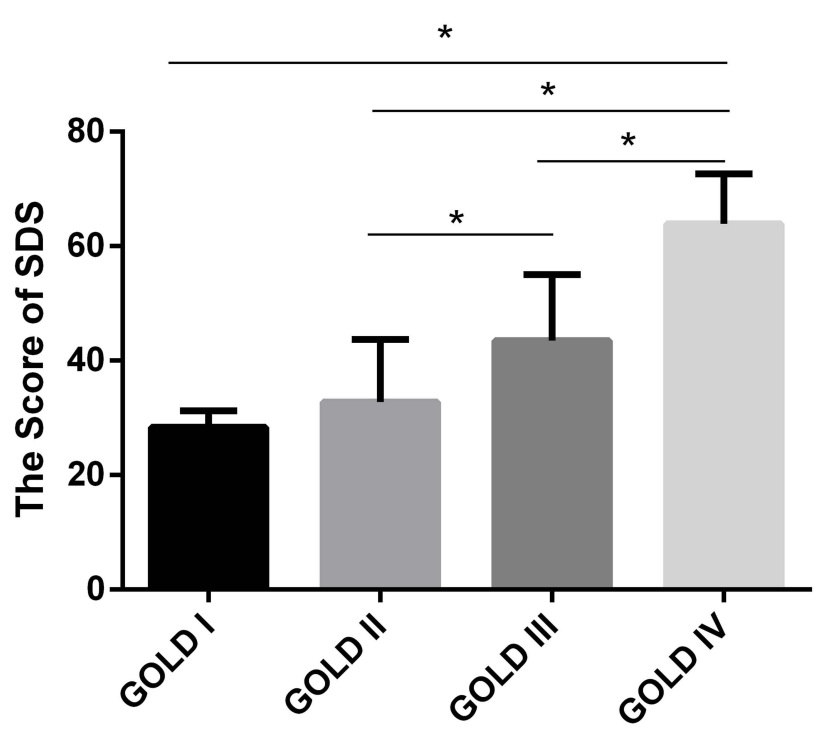

Figure 3 SDS score for different GOLD stages. The SDS score increased with disease severity. The average SDS score of patients at GOLD stages I to IV was $28.33 \pm 2.89,32.73 \pm 10.96,43.50 \pm \mathrm{II} .5 \mathrm{I}$, and $63.9 \mathrm{I} \pm 8.76$, respectively. *Means $P<0.05$.

Abbreviations: SDS, Self-Rating Depression Scale; GOLD, Global Initiative for Chronic Obstructive Lung Disease.

CODEXS vs CODEX 0.88 [0.82], $P=0.02$ [Figure 5B]; BODES vs BODE 0.83 [0.79], $P=0.03$ [Figure 5C]; BODEXS vs BODE 0.85 [0.79], $P=0.004$ [Figure 5D]). The cut-off point for all the multidimensional indices to differentiate frequent exacerbators was 3.5. ROC comparison showed that CODEXS had a better capacity to predict exacerbations than ADOS $(P=0.005)$, BODES $(P=0.01)$, and BODEXS $(P=0.007)$ (Figure 6).

\section{Validation of Multidimensional Indices}

In order to validate these new indices, we recruited 107 patients with COPD from the First Affiliated People's Hospital of Shaoyang College. The demographic characteristics are shown in Table 1. No significant statistic changes of the variables were found between the two

Table 3 Predictive Value of Different COPD Multivariate Indices for Frequent Exacerbators

\begin{tabular}{|l|l|l|l|l|l|}
\hline Index & OR & 95\% CI & AUC & $95 \% \mathbf{C I}$ & $\boldsymbol{P}$ \\
\hline CODEX & 2.79 & $1.83-4.26$ & 0.82 & $0.74-0.90$ & $<0.001$ \\
ADO & 2.0 & $1.38-2.91$ & 0.71 & $0.61-0.81$ & $<0.001$ \\
BODEX & 2.17 & $1.55-3.04$ & 0.79 & $0.70-0.87$ & $<0.001$ \\
BODE & 1.91 & $1.42-2.57$ & 0.79 & $0.70-0.87$ & $<0.001$ \\
\hline
\end{tabular}

Abbreviations: CODEX, comorbidities, airflow obstruction, dyspnea, previous severe exacerbation; ADO, age, dyspnea, airflow obstruction; BODE, body mass index, airflow obstruction, dyspnea, exercise; BODEX, body mass index, airflow obstruction, dyspnea, previous severe exacerbation; OR, odds ratio; $\mathrm{Cl}$, confidence interval; $A \cup C$, area under the curve. 


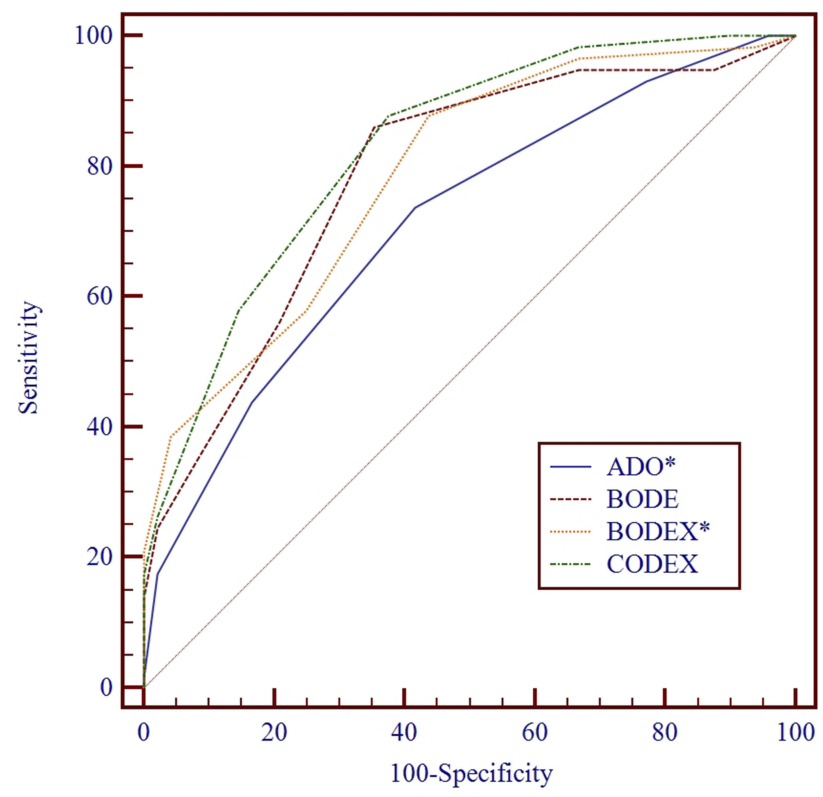

Figure 4 ROC comparison between previously established multidimensional indices for predicting frequent exacerbators. CODEX had a better predictive capacity for frequent exacerbators compared with ADO $(P=0.008)$ and BODEX $(P=0.03)$; the AUC were $0.82,0.71$, and 0.79 , respectively. $*$ Means $P<0.05$. Abbreviations: ROC, receiver operating characteristic; CODEX, comorbidities, airflow obstruction, dyspnea, previous severe exacerbation; ADO, age, dyspnea, airflow obstruction; BODE, body mass index, airflow obstruction, dyspnea, exercise; BODEX, body mass index, airflow obstruction, dyspnea, previous severe exacerbation; AUC, area under the curve.

cohorts of patients. After 1 year of follow-up, 64 patients had frequent exacerbations (59.81\%). Taking 3.5 as the cut-off point for CODEXS, BODEXS, and ADOS, the sensitivity to identify frequent exacerbators was $85.94 \%$, $82.81 \%$, and $84.38 \%$, respectively, and the specificity was $76.74 \%, 60.47 \%$, and $53.49 \%$, respectively (Table 5).

\section{Discussion}

In this study, we found depression was an independent risk factor for frequent exacerbation in patients with COPD.

Table 4 Predictive Value of Updated COPD Multivariate Indices for Frequent Exacerbators

\begin{tabular}{|l|l|l|l|l|l|}
\hline Index & OR & $\mathbf{9 5 \%} \mathbf{C l}$ & AUC & $\mathbf{9 5 \%} \mathbf{~ C l}$ & $\mathbf{P}$ \\
\hline CODEXS & 2.91 & $1.89-4.48$ & 0.88 & $0.8 I-0.94$ & $<0.001$ \\
ADOS & 1.97 & $1.48-2.63$ & 0.80 & $0.72-0.89$ & $<0.001$ \\
BODEXS & 2.15 & $1.58-2.94$ & 0.85 & $0.78-0.92$ & $<0.001$ \\
BODES & 1.80 & $1.4 I-2.30$ & 0.83 & $0.75-0.90$ & $<0.001$ \\
\hline
\end{tabular}

Abbreviations: CODEXS, comorbidities, airflow obstruction, dyspnea, previous severe exacerbation, depression; ADOS, age, dyspnea, airflow obstruction, depression; BODES, body mass index, airflow obstruction, dyspnea, exercise, depression; BODEXS, body mass index, airflow obstruction, dyspnea, previous severe exacerbation, depression; $\mathrm{OR}$, odds ratio; $\mathrm{Cl}$, confidence interval; $\mathrm{AUC}$, area under the curve.
The combination of SDS score with the existing indices BODE, BODEX, CODEX, and ADO improved the predictive value for frequent exacerbators significantly. We named these new indices BODES, BODEXS, CODEXS, and ADOS. We found CODEXS was superior to the other three multidimensional indices, and validated the finding in another cohort of patients with COPD. To our knowledge, no multicomponent indices took depression into consideration in previous studies. In our study, we developed and validated a new multidimensional index to predict frequent exacerbators among patients with COPD, which can be easily applied to clinical practice.

Depression is the leading cause of increased disability and impaired quality of life in older people worldwide. Many studies have reported a high rate of depression among patients suffering from COPD. ${ }^{16,24,25}$ The prevalence of depression in COPD varies from $6 \%$ to $57 \%$ due to different sample size, age group, and depression screening tool. ${ }^{26}$ There are many questionnaires used to study depression in COPD patients, including the Center for Epidemiologic Studies Depression Scale (CES-D), Geriatric Depression Scale (GDS), Hospital Anxiety and Depression Scale (HADS), SDS, and the Structured Clinical Interview for DSM-I. CES-D was designed to cover most depression symptoms, with emphasis on affective components; GDS was designed for screening depression in older populations, HADS was designed for screening depression in the general medical population, and SDS can screen for depression, as well as classify its severity. ${ }^{27}$ In our study, we wanted to include the SDS score in a multidimensional index, so we chose SDS to assess the severity of depression and assigned a different value, ranging from 0 to 4 based on the SDS score, to the previously reported indices BODE, BODEX, ADO, and CODEX. We found the percentage of COPD patients with depression was $25.71 \%$, similar to a previous study. ${ }^{28}$ The prevalence of depression $(35.09 \%)$ was much higher in frequent exacerbators compared with infrequent exacerbators $(12.50 \%)$, indicating that depression is related to exacerbations in COPD patients. More severe depression was found in patients at a higher GOLD stage. A possible reason for this result may be that COPD and depression are deeply interrelated. It is well known that depression plays a role in the initiation and maintenance of smoking, that smoking is a critical risk factor for the development of COPD, and that COPD, in turn, contributes to the worsening of depression. $^{29,30}$

Through logistic regression analysis, we found that depression is an independent risk factor for predicting 

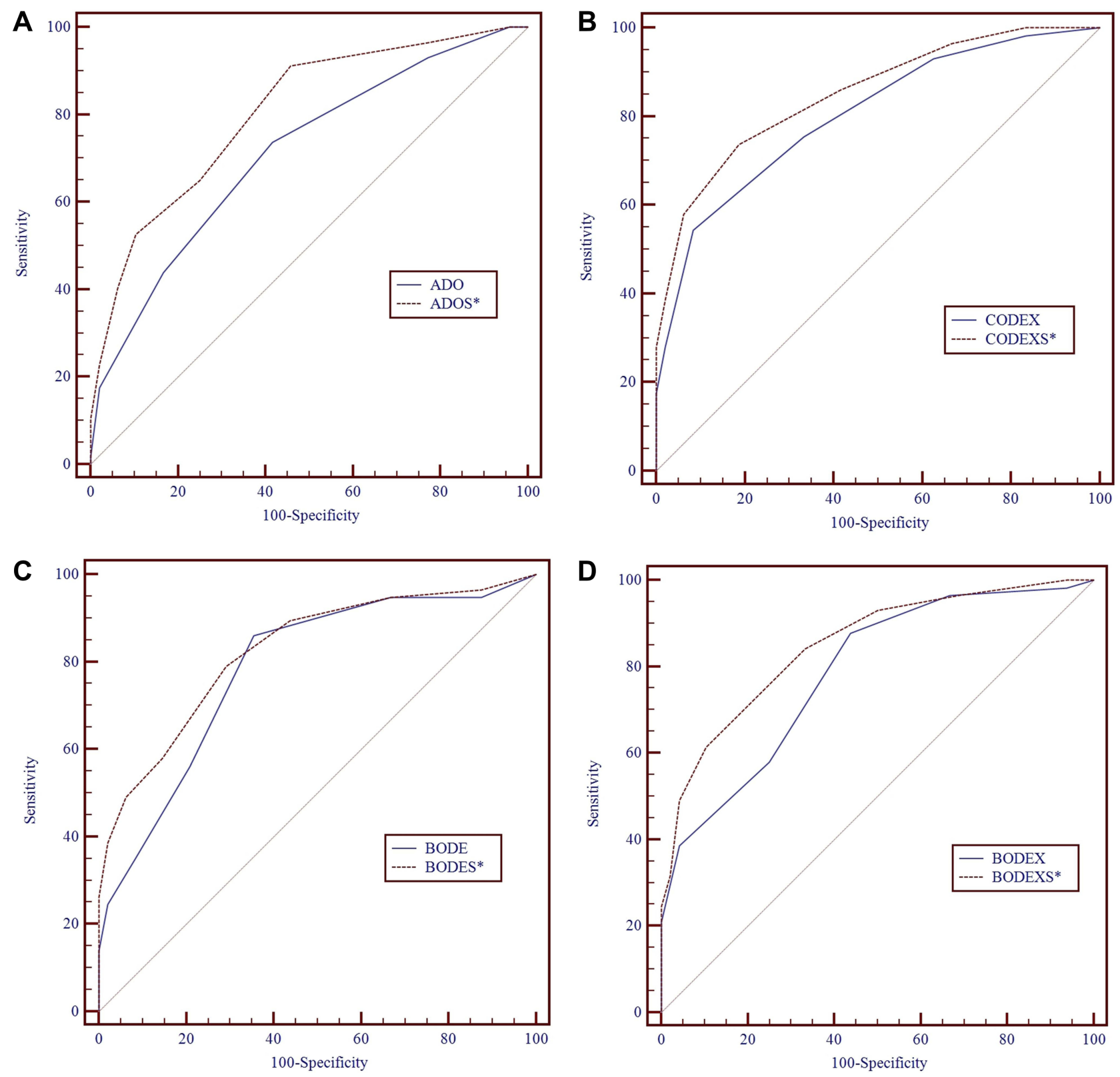

Figure 5 ROC comparison between updated multidimensional indices and previous indices. The updated multidimensional indices improved the predictive capacity of all the previous indices (A) (ADOS vs ADO, 0.80 [0.7I], $P=0.0002 ;(B)$ CODEXS vs CODEX 0.88 [0.82], $P=0.02 ;($ C) BODES vs BODE 0.83 [0.79], $P=0.03 ;(D)$ BODEXS vs BODEX 0.85 [0.79], $P=0.004)$. *Means $P<0.05$.

Abbreviations: ROC, receiver operating characteristic; ADOS, age, dyspnea, airflow obstruction, depression; ADO, age, dyspnea, airflow obstruction; CODEXS, comorbidities, airflow obstruction, dyspnea, previous severe exacerbation, depression; CODEX, comorbidities, airflow obstruction, dyspnea, previous severe exacerbation; BODES, body mass index, airflow obstruction, dyspnea, exercise, depression; BODE, body mass index, airflow obstruction, dyspnea, exercise; BODEXS, body mass index, airflow obstruction, dyspnea, previous severe exacerbation, depression; BODEX, body mass index, airflow obstruction, dyspnea, previous severe exacerbation.

frequent exacerbators among COPD patients. To determine whether taking depression into consideration could improve the predictive value of a multidimensional index, we converted the SDS score into a 5-point scale, ranging from 0 to 4, representing 25-39, 40-49, 50-59, 60-69, and $\geq 70$, respectively. The rationale for setting up this scale was based on the severity of depression. Index scores of 25 to 49 indicate no depression, 50-59 indicates mild depression, 60-69 indicates moderate depression, and scores $\geq 70$ indicate severe depression; ${ }^{18} 0-1$ represents no depression, and 2-4 represent mild, moderate, and severe depression, respectively.

The most widely recognized variables associated with exacerbations in patients with $\mathrm{COPD}$ are $\mathrm{FEV}_{1} \%$, dyspnea, and exacerbations in the previous year. ${ }^{31-33}$ The first two are the basis of multicomponent indices; the number of 


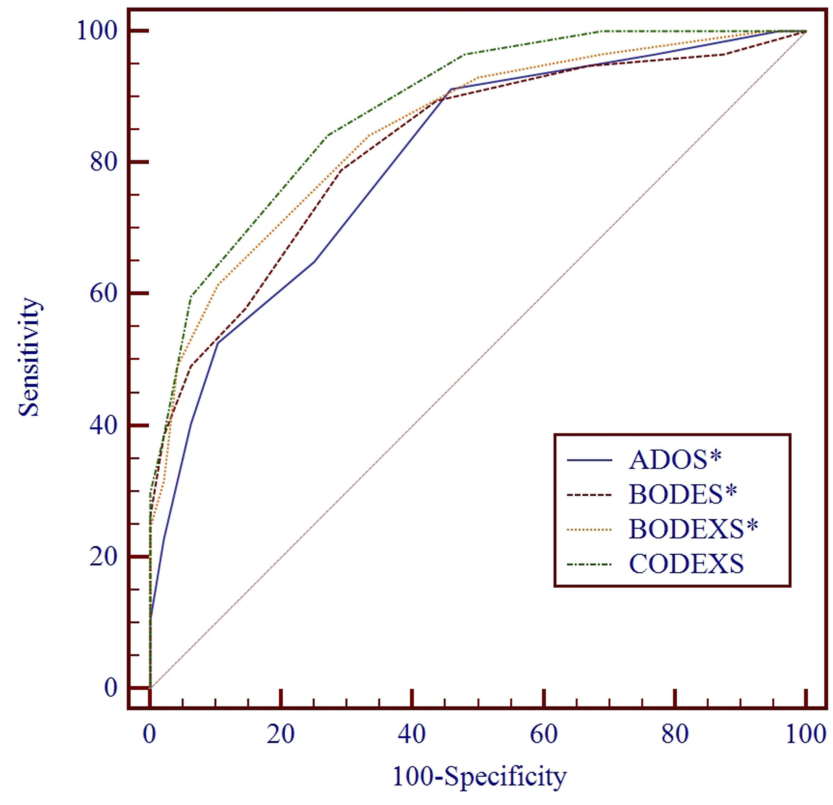

Figure 6 ROC comparison between updated multidimensional indices for predicting frequent exacerbators. CODEXS had better a capacity to predict exacerbations than ADOS $(P=0.005)$, BODES $(P=0.01)$, and BODEXS $(P=0.007)$. $*$ Means $P<$ 0.05 .

Abbreviations: ROC, receiver operating characteristic; CODEXS, comorbidities, airflow obstruction, dyspnea, previous severe exacerbation, depression; ADOS, age, dyspnea, airflow obstruction, depression; BODES, body mass index, airflow obstruction, dyspnea, exercise, depression; BODEXS, body mass index, airflow obstruction, dyspnea, previous severe exacerbation, depression.

exacerbations in the previous year has been proved to be an independent risk factor, and some indices have also included this factor. Other factors, such as age, comorbidities, BMI, and 6-mins walk test, have also been used to develop some multicomponent indices. Several multicomponent indices are widely recognized, including CODEX, BODEX, ADO, and BODE. In this study, we demonstrated that all these indices could predict 1-year exacerbations in COPD patients, which was similar to previous studies. ${ }^{11,34-37}$ ROC comparison analysis showed that CODEX was superior to BODEX, ADO, and BODE for

Table 5 Validation of Multicomponent Indices

\begin{tabular}{|l|l|l|l|l|l|l|l|}
\hline \multirow{2}{*}{$\begin{array}{l}\text { Follow-Up } \\
\text { Results }\end{array}$} & \multicolumn{2}{|c|}{ CODEXS } & \multicolumn{2}{|c|}{ BODEXS } & \multicolumn{2}{|c|}{ ADOS } & \multirow{2}{*}{ Total } \\
\cline { 2 - 7 } & + & - & + & - & + & - & \\
\hline+ & 55 & 9 & 53 & 11 & 54 & 10 & 64 \\
- & 10 & 33 & 17 & 26 & 20 & 23 & 43 \\
Total & 65 & 42 & 70 & 37 & 74 & 33 & 107 \\
\hline
\end{tabular}

Note: +Means frequent exacerbators; -Means infrequent exacerbators.

Abbreviations: CODEXS, comorbidities, airflow obstruction, dyspnea, previous severe exacerbation, depression; ADOS, age, dyspnea, airflow obstruction, depression; BODEXS, body mass index, airflow obstruction, dyspnea, previous severe exacerbation, depression. predicting 1-year exacerbation. CODEX was first proposed by Pedro Almagro; it was an evolution of the BODE and BODEX indices, retaining their cut-offs for dyspnea, obstruction, and previous exacerbations, but replacing BMI with comorbidity measured by the original Charlson index modified by age. Although low BMI has been proved to be associated with a poor survival, the Charlson index shows a better prognostic capacity. ${ }^{11}$ This may be why CODEX showed a better capacity for predicting frequent exacerbators.

The importance of depression in COPD is well established. However, to date, none of the COPD indices have included depression as a prognostic variable. In our study, we found that SDS score was correlated to symptoms and airflow obstruction, and confirmed that adding the SDS value into previous multicomponent indices improved their predictive value for frequent exacerbators significantly. Similar to CODEX, CODEXS is the most useful, with the highest sensitivity and specificity. As such, clinicians should pay more attention to those who are judged to be frequent exacerbators, making proper clinical interventions.

Our study has several limitations. Firstly, the population for validating the indices came from the same hospital in different time periods with the same inclusion and exclusion criteria; it would be better to validate these indices with a multicenter study, and it is worth having a longer follow-up period to assess the predictive value of CODEXS for the long-term outcome. Secondly, our study had a clear predominance of men. But this finding is similar to all studies of COPD performed in our area and it is probably to the fact that there are a higher proportion of men to smoke than that in women. ${ }^{20,21,38}$ Finally, although we found that depression is an independent risk factor for exacerbation, we did not treat the patients with antidepressant or psychological intervention; in a future study, it would be worth determining whether proper psychological intervention improves the prognosis of patients with COPD.

\section{Conclusion}

In conclusion, depression is an independent risk factor for COPD exacerbations. CODEXS is a simple, effective tool for predicting the risk of COPD exacerbation.

\section{Abbreviations}

ADO, age, dyspnea, airflow obstruction; ADOS, age, dyspnea, airflow obstruction, depression; AECOPD, acute exacerbations of chronic obstructive pulmonary disease; AUC, area under the curve; BMI, body mass 
index; BODE, body mass index, airflow obstruction, dyspnea, exercise; BODES, body mass index, airflow obstruction, dyspnea, exercise, depression; BODEX, body mass index, airflow obstruction, dyspnea, previous severe exacerbation; BODEXS, body mass index, airflow obstruction, dyspnea, previous severe exacerbation, depression; CAT, COPD assessment test; CCQ, Clinical COPD Questionnaire; CES-D, Center for Epidemiologic Studies Depression Scale; CI, confidence interval; CODEX, comorbidities, airflow obstruction, dyspnea, previous severe exacerbation; CODEXS, comorbidities, airflow obstruction, dyspnea, previous severe exacerbation, depression; COPD, chronic obstructive pulmonary disease; $\mathrm{FEV}_{1}$, forced expiratory volume in 1 second; FVC, forced vital capacity; GDS, Geriatric Depression Scale; GOLD, Global Initiative of Chronic Obstructive Lung Disease; HADS, Hospital Anxiety and Depression Scale; mMRC, modified Medical Research Council Dyspnea Scale; OR, odds ratio; ROC, receiver operating characteristic; SD, standard deviation; SDS, Self-Rating Depression Scale.

\section{Data Sharing Statement}

The authors confirm that the data supporting the findings of this study are available within the article. The datasets used and/or analyzed during the current study are available from the corresponding author on reasonable request, the authors will not share any individual deidentified participant date or other relevant study documents.

\section{Ethics Approval and Consent to Participate}

The research protocol was approved by the local Ethics Committee of the First Affiliated People's Hospital of Shaoyang College (number: C2016123) and conducted in accordance with the Declaration of Helsinki and its amendments. All subjects gave written informed consent to participate in the study.

\section{Acknowledgments}

This study was funded by grants from the Fundamental Research Funds for the Central Universities of Central South University (2017zzts228 to Doctor Aiyuan Zhou). We want to thank Dr. Alexandra Racanelli (Division of Pulmonary and Critical Care Medicine, Weill Cornell Medical College) for proofreading the manuscript.
Dingding Deng and Aiyuan Zhou are co-first authors for this study.

\section{Author Contributions}

All authors contributed to data analysis, drafting and revising the article, gave final approval of the version to be published, and agree to be accountable for all aspects of the work.

\section{Disclosure}

The authors report no conflicts of interest in this work.

\section{References}

1. Global Initiative for Chronic Obstructive Lung Disease(GOLD). Global strategy for the diagnosis, management, and prevention of chronic obstructive pulmonary disease; 2019. Available from: http:// goldcopd.org/. Accessed January 22, 2020.

2. Makris D, Moschandreas J, Damianaki A, et al. Exacerbations and lung function decline in COPD: new insights in current and ex-smokers. Respir Med. 2007;101:1305-1312. doi:10.1016/j.rmed.2006.10.012

3. Donaldson GC, Seemungal TA, Bhowmik A, Wedzicha JA. Relationship between exacerbation frequency and lung function decline in chronic obstructive pulmonary disease. Thorax. 2002;57:847-852. doi:10.1136/thorax.57.10.847

4. Byng D, Lutter JI, Wacker ME, et al. Determinants of healthcare utilization and costs in COPD patients: first longitudinal results from the German COPD cohort COSYCONET. Int J Chron Obstruct Pulmon Dis. 2019;14:1423-1439. doi:10.2147/COPD.S201899

5. Soler-Cataluna JJ, Martinez-Garcia MA, Roman Sanchez P, et al. Severe acute exacerbations and mortality in patients with chronic obstructive pulmonary disease. Thorax. 2005;60:925-931. doi:10.1136/thx.2005.040527

6. Bafadhel M, McKenna S, Terry S, et al. Acute exacerbations of chronic obstructive pulmonary disease: identification of biologic clusters and their biomarkers. Am J Respir Crit Care Med. 2011;184:662-671. doi:10.1164/rccm.201104-0597OC

7. Zhou A, Zhou Z, Zhao Y, Chen P. The recent advances of phenotypes in acute exacerbations of COPD. Int J Chron Obstruct Pulmon Dis. 2017;12:1009-1018. doi:10.2147/COPD

8. Hurst JR, Vestbo J, Anzueto A, et al. Susceptibility to exacerbation in chronic obstructive pulmonary disease. $N$ Engl $J$ Med. 2010;363:1128-1138. doi:10.1056/NEJMoa0909883

9. Nishimura K, Izumi T, Tsukino M, Oga T. Dyspnea is a better predictor of 5-year survival than airway obstruction in patients with COPD. Chest. 2002;121:1434-1440. doi:10.1378/chest.121.5.1434

10. Vestbo J, Hurd SS, Agusti AG, et al. Global strategy for the diagnosis, management, and prevention of chronic obstructive pulmonary disease: GOLD executive summary. Am J Respir Crit Care Med. 2013;187:347-365. doi:10.1164/rccm.201204-0596PP

11. Almagro P, Soriano JB, Cabrera FJ, et al. Short- and medium-term prognosis in patients hospitalized for COPD exacerbation: the CODEX index. Chest. 2014;145:972-980. doi:10.1378/chest.13-1328

12. Celli BR, Cote CG, Marin JM, et al. The body-mass index, airflow obstruction, dyspnea, and exercise capacity index in chronic obstructive pulmonary disease. $N$ Engl J Med. 2004;350:1005-1012. doi:10.1056/NEJMoa021322

13. Puhan MA, Garcia-Aymerich J, Frey M, et al. Expansion of the prognostic assessment of patients with chronic obstructive pulmonary disease: the updated BODE index and the ADO index. Lancet. 2009;374:704-711. doi:10.1016/S0140-6736(09)61301-5 
14. van Manen JG, Bindels PJ, Dekker FW, et al. Risk of depression in patients with chronic obstructive pulmonary disease and its determinants. Thorax. 2002;57:412-416. doi:10.1136/thorax.57.5.412

15. Di Marco F, Verga M, Reggente M, et al. Anxiety and depression in COPD patients: the roles of gender and disease severity. Respir Med. 2006;100:1767-1774. doi:10.1016/j.rmed.2006.01.026

16. Quint JK, Baghai-Ravary R, Donaldson GC, Wedzicha JA. Relationship between depression and exacerbations in COPD. Eur Respir J. 2008;32:53-60. doi:10.1183/09031936.00120107

17. Zung WW. A Self-Rating Depression Scale. Arch Gen Psychiatry. 1965;12:63-70. doi:10.1001/archpsyc.1965.01720310065008

18. Zung WW. From art to science. The diagnosis and treatment of depression. Arch Gen Psychiatry. 1973;29:328-337. doi:10.1001/ archpsyc.1973.04200030026004

19. Quan H, Li B, Couris CM, et al. Updating and validating the Charlson comorbidity index and score for risk adjustment in hospital discharge abstracts using data from 6 countries. Am J Epidemiol. 2011;173:676-682. doi:10.1093/aje/kwq433

20. Zhou A, Zhou Z, Peng Y, et al. The role of CAT in evaluating the response to treatment of patients with AECOPD. Int J Chron Obstruct Pulmon Dis. 2018;13:2849-2858. doi:10.2147/COPD.S175085

21. Zhou Z, Zhou A, Zhao Y, Duan J, Chen P. A comparison of the assessment of health status between CCQ and CAT in a Chinese COPD clinical population: a cross-sectional analysis. Int J Chron Obstruct Pulmon Dis. 2018;13:1675-1682. doi:10.2147/COPD

22. Laboratories ATSCoPSfCPF. ATS statement: guidelines for the six-minute walk test. Am J Respir Crit Care Med. 2002;166:111-117. doi:10.1164/ajrccm.166.1.at1102

23. Espantoso-Romero M, Roman Rodriguez M, Duarte-Perez A, et al. External validation of multidimensional prognostic indices (ADO, BODEx and DOSE) in a primary care international cohort (PROEPOC/COPD cohort). BMC Pulm Med. 2016;16:143. doi:10.1186/s12890-016-0305-2

24. Mikkelsen RL, Middelboe T, Pisinger C, Stage KB. Anxiety and depression in patients with chronic obstructive pulmonary disease (COPD). A review. Nord J Psychiatry. 2004;58:65-70. doi:10.1080/ 08039480310000824

25. Yohannes AM, Alexopoulos GS. Depression and anxiety in patients with COPD. Eur Respir Rev. 2014;23:345-349. doi:10.1183/ 09059180.00007813

26. Zhang MW, Ho RC, Cheung MW, Fu E, Mak A. Prevalence of depressive symptoms in patients with chronic obstructive pulmonary disease: a systematic review, meta-analysis and meta-regression. Gen Hosp Psychiatry. 2011;33:217-223. doi:10.1016/j.genhosppsych.2011.03.009
27. Matte DL, Pizzichini MM, Hoepers AT, et al. Prevalence of depression in COPD: a systematic review and meta-analysis of controlled studies. Respir Med. 2016;117:154-161. doi:10.1016/j.rmed.2016.06.006

28. Hanania NA, Mullerova H, Locantore NW, et al. Determinants of depression in the ECLIPSE chronic obstructive pulmonary disease cohort. Am J Respir Crit Care Med. 2011;183:604-611. doi:10.1164/ rccm.201003-0472OC

29. Norwood R. Prevalence and impact of depression in chronic obstructive pulmonary disease patients. Curr Opin Pulm Med. 2006;12:113-117. doi:10.1097/01.mcp.0000208450.50231.c6

30. Schneider C, Jick SS, Bothner U, Meier CR. COPD and the risk of depression. Chest. 2010;137:341-347. doi:10.1378/chest.09-0614

31. Zider AD, Wang X, Buhr RG, et al. Reduced COPD exacerbation risk correlates with improved FEV1: a meta-regression analysis. Chest. 2017;152:494-501. doi:10.1016/j.chest.2017.04.174

32. Garcia-Gutierrez S, Quintana JM, Unzurrunzaga A, et al. Predictors of change in dyspnea level in acute exacerbations of COPD. COPD. 2016;13:303-311. doi:10.3109/15412555.2015.1078784

33. Guerra B, Gaveikaite V, Bianchi C, Puhan MA. Prediction models for exacerbations in patients with COPD. Eur Respir Rev. 2017;26:160061.

34. Almagro P, Martinez-Camblor P, Miravitlles M, et al. External validation and recalculation of the CODEX index in COPD patients. A 3CIAplus Cohort study. COPD. 2019;16:8-17. doi:10.1080/ 15412555.2018.1484440

35. Marin JM, Carrizo SJ, Casanova C, et al. Prediction of risk of COPD exacerbations by the BODE index. Respir Med. 2009;103:373-378. doi:10.1016/j.rmed.2008.10.004

36. Chen $\mathrm{CZ}$, Ou CY, Yu CH, et al. Comparison of global initiative for chronic obstructive pulmonary disease 2013 classification and body mass index, airflow obstruction, dyspnea, and exacerbations index in predicting mortality and exacerbations in elderly adults with chronic obstructive pulmonary disease. J Am Geriatr Soc. 2015;63:244-250. doi:10.1111/jgs. 13258

37. Jones RC, Price D, Chavannes NH, et al. Multi-component assessment of chronic obstructive pulmonary disease: an evaluation of the ADO and DOSE indices and the global obstructive lung disease categories in international primary care data sets. NPJ Prim Care Respir Med. 2016;26:16010. doi:10.1038/npjpcrm.2016.10

38. Zhou A, Luo L, Liu N, et al. Prospective development of practical screening strategies for diagnosis of asthma-COPD overlap. Respirology. Epub 2019 Nov 27.

International Journal of Chronic Obstructive Pulmonary Disease

Dovepress

\section{Publish your work in this journal}

The International Journal of COPD is an international, peer-reviewed journal of therapeutics and pharmacology focusing on concise rapid reporting of clinical studies and reviews in COPD. Special focus is given to the pathophysiological processes underlying the disease, intervention programs, patient focused education, and self management protocols. This journal is indexed on PubMed Central, MedLine and CAS. The manuscript management system is completely online and includes a very quick and fair peer-review system, which is all easy to use. Visit http://www.dovepress.com/testimonials.php to read real quotes from published authors. 